Schlegel, M. (2017). Kriterien wissenschaftlich begründeter Psychotherapie und Aspekte ihrer emanzipierenden säkularen Spiritualität. Psychotherapie-Wissenschaft, 7(1), 45-56.

Schulthess, P. (2015). Psychotherapie gehört abgegrenzt von der Transpersonalen Psychologie und Esoterik. à jour! PsychotherapieBerufsentwicklung, 1, 23-26.

Utsch, M., Anderssen-Reuster, U., Frick, E., Gross, W., Murken, S., Schouler-Ocak, M., Stotz-Ingenlath, G. (2017). Empfehlungen zum Umgang mit Religiosität und Spiritualität in Psychiatrie und Psychotherapie. Spiritual Care, 6(1), 141-146.

\section{Jens Tasche und Reinhard Weber-Steinbach (Hrsg.). (2018). Bioenergetik als mentalisierende Körperpsychotherapie}

\author{
Göttingen: Vandenhoeck \& Ruprecht. \\ ISBN: 978-3-6474-5197-8. \\ 226 Seiten. 30,00 EUR, 44,90 CHF
}

Psychotherapie-Wissenschaft 9 (1) 85-86 2019 www.psychotherapie-wissenschaft.info CC BY-NC-ND

https://doi.org/10.30820/1664-9583-2019-1-85

In dem von Jens Tasche und Reinhard Weber-Steinbach herausgegebenen Buch Bioenergetik als mentalisierende Körperpsychotherapie unternehmen neun (sieben davon bioenergetisch ausgebildete) Psychotherapeut/innen den Versuch eines Brückenschlags zwischen bioenergetischer Analyse und mentalisierungsbasierter Therapie. Im Kontext aktueller Bemühungen, rivalisierendes Schulendenken zu überwinden, darf das als sehr begrüssenswertes Unterfangen angesehen werden. Während Alexander Lowen, (Mit-)Begründer der Bioenergetischen Analyse, seinen Schülern immer wieder empfahl: «Get out of your head, get into your body!», empfehlen die Autor/innen des vorliegenden Buches das Umgekehrte: «Vergesst nicht, dass Ihr einen Kopf habt. Nutzt Eure kognitiven Fähigkeiten, um Euch Vorstellungen/Modelle von Eurem eigenen Erleben und Verhalten wie auch dem Eurer Mitmenschen zu machen, um zu verstehen und empathisch mit Euch selbst und anderen umzugehen.»

Eiligen Leser/innen wird es allerdings bis zum Schluss des Buches nicht leicht gemacht, den Begriff des Mentalisierens wie auch einzelne Konzepte der Bioenergetischen Analyse konzise zu erfassen, geschweige denn, den Sprachgebrauch der einen oder der anderen Methode vergleichend aufeinander zu beziehen. Die Autor/innen des Buches unternehmen ihre ganz persönlichen Herleitungen aus der Literatur und ziehen ihre je unterschiedlichen Schlussfolgerungen. Die Leser/innen sehen sich also mit höchst heterogenen Texten konfrontiert. Das mag Ergebnissen empirischer Forschungsarbeiten entsprechen, wonach vor allem die behandelten Patient/innen, in zweiter Linie die behandelnden Psychotherapeut/innen und praktisch überhaupt nicht die zur Anwendung gebrachte Methode (bzw. die Methode, der die Therapeut/innen anhängen) voraussagt, was in einer konkreten Behandlungssitzung geschieht (z. B. Koemeda-Lutz et al., 2016).
Schultz-Venrath definiert in seinem Vorwort «Mentalisieren als prozessuales Regulationsmodell früher Körperund Affekterfahrungen» (S. 13) und versteht Mentalisieren als «unspezifischen Wirkfaktor», der "wahrscheinlich in den meisten Psychotherapien und Körperpsychotherapien» zur Anwendung kommt (S. 16).

Reinhard Weber-Steinbach sieht die Wurzeln des Mentalisierungskonzeptes in psychoanalytischen Intersubjektivitätskonzepten, wonach das individuelle Selbst in einem interaktiven Zwischenraum entstehe. Leider setzt er diese von ihm so benannte dritte Topik der Psychoanalyse nicht zu den vor allem von Kolleg/innen in Kalifornien in den 1970er Jahren vorangetriebenen relationalen Ansätzen in der Bioenergetischen Analyse (R. Hilton, V. Wink Hilton, H. Resneck-Sannes) in Beziehung. Er referiert ein Entwicklungsmodell, das auf die affektiv-interaktive Qualität der Primärbeziehungen setzt und hebt das Verdienst von Fonagy und Mitarbeiter/innen hervor, die Bindungstheorie und das Theory of Mind (TOM) Konzept in die Psychoanalyse integriert zu haben.

Am Beispiel von klinischen Problemstellungen zeichnet Alice Moll ihren eigenen Weg als Bioenergetische Analytikerin zur Integration des Mentalisierungskonzeptes nach. Sie referiert die Entwicklungsstufen zur Mentalisierungsfähigkeit (teleologischer, Äquivalenz- und Als-Ob-Modus). Die Fähigkeit zu mentalisieren kann unter krisenhaften oder traumatischen Bedingungen zusammenbrechen oder, falls diese in der Kindheit auftreten, eine Ausbildung dieser Fähigkeit verhindern und so zu Strukturdefiziten führen.

Steve Hofmann plädiert dafür, das Mentalisierungskonzept explizit in körperpsychotherapeutische Ausbildungen zu integrieren.

Marion Baum versucht einen Brückenschlag zwischen Embodiment und Mentalisierungskonzepten.

Im «Interview» mit Christiane Reepen-Bading, Jens Tasche und Reinhard Weber-Steinbach wird die Bedeutung der Mentalisierungsfähigkeit in der psychosexuellen Entwicklung beleuchtet und diskutiert.

Eine ausführliche und interessante Falldarstellung liefert Barbara Antonowicz-Wlasinska. Sie beschreibt eine Patientin mit einer tiefen strukturellen Störung, der sie «eine Kombination aus einer affektfokussierten Körperpsychotherapie» und einer mentalisierenden Gesprächspsychotherapie angedeihen lässt. In ersterer soll die Patientin lernen, «die eigenen (positiven und negativen) Empfindungen, Sehnsüchte und Bedürfnisse zu fühlen, zu ertragen und zur Grundlage eines authentischen Selbsterlebens zu machen; letztere soll ihr ermöglichen, das Gefühlte zu reflektieren und zu symbolisieren»(S. 161f.).

Carsten Holle schreibt über Integrationsbemühungen eines bioenergetischen und eines mentalisierungsbasierten Ansatzes in der Kinder- und Jugendpsychiatrie in Neuruppin.

Jens Tasche formuliert acht Thesen zu Übereinstimmungen und Unterschieden zwischen Bioenergetischer Analyse und neueren Ansätzen in der Psychoanalyse (exemplarisch abgehandelt am Mentalisierungskonzept von Fonagy et al., an der Neuropsychoanalyse von A. N. Schore und am intersubjektiven Ansatz von M. Ermann). 
Sehr interessant, und mich persönlich am meisten nachdenklich stimmend, fand ich das Abschlusskapitel von Martin Herberhold, der über mögliche Auswirkungen der in den Industrienationen fortschreitenden Digitalisierung auf unsere psychotherapeutische Tätigkeit und das Selbstverständnis von Psychotherapeut/innen nachdenkt und schreibt.

Insgesamt illustriert auch das vorliegende Buch den in der Psychotherapieforschung vielfach publizierten Befund, dass einzelne Psychotherapeut/innen ihre Ausbildungen in einer bestimmten Psychotherapiemethode so lange um weitere Konzepte und Techniken (mehr oder weniger eklektisch) erweitern, bis sie sich den Problemstellungen im klinischen Alltag einigermassen gewachsen fühlen.

Das Buch sei allen, denen bisher der Körper in der Psychoanalyse gefehlt hat, und denen, die in Körperpsychotherapien einen gewissen Mangel an kognitiver Raffinesse vermisst haben, empfohlen.

Margit Koemeda

\section{Frank-M. Staemmler (2017). Relationalität in der Gestalttherapie: Kontakt und Verbundenheit}

\author{
Gevelsberg: EHP. ISBN: 978-3-89797-103-5. \\ 292 Seiten. 26,99 EUR, 41,99 CHF
}

\author{
Psychotherapie-Wissenschaft 9 (1) 86-87 2019 \\ www.psychotherapie-wissenschaft.info \\ CC BY-NC-ND \\ https://doi.org/10.30820/1664-9583-2019-1-86
}

Gleich zu Beginn stellt Frank-M. Staemmler klar, was er mit dieser Neuerscheinung beabsichtigt:

«Ich verstehe den vorliegenden Text eher als ein Zusammenführen und eine Fortsetzung früherer Gedankengänge. Ich knüpfe dabei hauptsächlich an die Inhalte dreier meiner Bücher an: Therapeutische Beziehung und Diagnose - Gestalttherapeutische Antworten (1993), Das Geheimnis des Anderen - Empathie in der Psychotherapie (2009a) und Das dialogische Selbst - Postmodernes Menschenbild und psychotherapeutische Praxis (2015a)» (S. 15).

Der gemeinsame Fokus ist die menschliche Relationalität. Mit Zitaten am Anfang von jedem Kapitel ehrt Staemmler den 1949 verstorbenen Hans Trüb, dessen posthum erschienenes Werk Heilung aus der Begegnung - Überlegungen zu einer dialogischen Psychotherapie einen Grundstein für die Relationalität in der Psychotherapie gelegt hat.

Staemmler folgt der historischen Spur des Paradigmenwechsels von einer "Ein-Person-Psychologie» (bei der Individualismus in den 1960er und 1970er Jahren im Vordergrund stand) zur «Zwei-Personen-Psychologie» und zwischenmenschlichen Wechselseitigkeit der sogenannten relationalen Wende (die 1980er Jahre). Was die Gestalttherapie betrifft, so hat die relationale Wende zwei
Schwerpunkte hervorgebracht. Der eine Schwerpunkt bezieht sich auf ethische Gesichtspunkte, die Gestalttherapeuten und Gestalttherapeutinnen sich zu Herzen nehmen sollen: sich möglichst um eine Ich-Du-Haltung im Sinne Martin Bubers in der therapeutischen Arbeit bemühen und die gemeinsame Suche nach Bedeutung, was die Aufgabe einer unangebrachten Haltung der Unfehlbarkeit seitens des Therapeuten bzw. der Therapeutin impliziert. Der zweite Schwerpunkt ist inhaltlicher Natur: Therapeuten und Therapeutinnen sollten sich nicht nur auf die individuellen psychologischen Prozesse ihrer Patienten und Patientinnen konzentrieren, sondern auch die eigenen Prozesse wie die zwischenmenschlichen Ereignisse, die sich in der Therapiestunde einstellen, sollen volle Aufmerksamkeit erhalten.

Staemmler plädiert dann für einen weiteren Paradigmenwechsel, vom «Selbst-in-Beziehung» zum «relationalen Selbst». "Schwache Relationalität», ein Begriff, den Staemmler von Brent Slife entlehnt, beschreibt Ersteres. «Selbst-in-Beziehung» kann man «als ein Individuum interpretieren, das primär aus sich selbst heraus existiert und dann, in einem zweiten Schritt, auf andere trifft, sich auf Beziehungen mit ihnen ein- und sich dann von ihnen beeinflussen lässt» (S. 175). Letzteres erweitert den Begriff von Relationalität, begreift Menschen als grundsätzlich aufeinander bezogen sowie miteinander verwoben und wird als «starke Relationalität» bezeichnet. Bezugnehmend auf Gestaltkollegen und Gestaltkolleginnen und andere Therapieschulen betont Staemmler wie Beziehungen und dynamische Wechselseitigkeiten Voraussetzungen für die Entwicklung des Selbst und der wahrnehmbaren Welt sind, nicht umgekehrt.

Das Buch ist historisch aufgebaut und Staemmler fügt, wie in früheren Publikationen, «Textboxen» mit zahlreichen Beispielen aus seiner Praxis oder passende theoretische Ergänzungen dem Haupttext hinzu. Die Fallvignetten zeigen einerseits exemplarisch wie Staemmlers Ansatz in der Praxis aussieht, andererseits machen sie deutlich, wie sehr er selbst von der Gegenseitigkeit in seinem Beruf als Psychotherapeut profitiert:
«Denn meine Tätigkeit als Psychotherapeut gestattet mir, einen großen Teil meines beruflichen Lebens in Verbundenheit mit anderen Menschen und in Fürsorge für sie zu verbringen. So sehr es von außen betrach- tet den Anschein haben mag, als engagierte ich mich hauptsächlich für meine Klienten, so sehr tue ich dabei ständig auch viel für mich selbst und für mein eigenes Wohlbefinden. Das Erleben von Resonanz und Verbun- denheit - ungeachtet der jeweiligen Einzig- und Anders- artigkeit meiner Klientinnen - sowie die Haltung der Gastlichkeit und Fürsorge sind dabei neben dem Erwerb von Anerkennung und der Erfahrung von Selbstwirk- samkeit die für mich maßgeblichen Faktoren» (S. 10).

Eine Ermutigung für Praktizierende, jung und alt, in einem Arbeitstag, der mit einer verwirrenden Mischung aus Glück, Betroffenheit und Erschöpfung endet, eine Quelle der Kreativität und Regenerierung zu finden. 